\title{
Multiscale features of density and frequency spectra from nonlinear gyrokinetics
}

\author{
T. Görler and F. Jenko \\ Max-Planck-Institut für Plasmaphysik, EURATOM Association, \\ Boltzmannstraße 2, D-85748 Garching, Germany
}

(Dated: December 18, 2008)

\begin{abstract}
Gyrokinetic turbulence simulations covering both electron and ion spatio-temporal scales selfconsistently are presented. It is found that for experimentally realistic transport levels at long wavelengths, electron temperature gradient modes may yield substantial or even dominant highwavenumber contributions to the electron heat flux. It is investigated in which way this behavior is reflected in several experimentally accessible quantities as for instance density or frequency spectra.
\end{abstract}

\section{INTRODUCTION}

While it is widely accepted that turbulent fluctuations are the physical origin of the anomalous transport observed in magnetized fusion plasmas, our present understanding of this nonlinear phenomenon is still rather fragmentary. One important open question concerns the role of high-wavenumber (i.e., sub-ion-gyroradius scale) contributions to the electron heat flux. Over the last several years, significant evidence has been collected in both dedicated experimental studies [1-5] and nonlinear gyrokinetic simulations [6-16] which suggests that electron temperature gradient (ETG) modes may yield substantial or even dominant high-wavenumber contributions to the electron heat transport under certain conditions, e.g., in plasmas with dominant electron heating, relatively high beta values, substantial equilibrium $\mathbf{E} \times \mathbf{B}$ shear, and (internal or edge) transport barriers.

This situation triggered a serious effort in the fusion community to extend existing experimental diagnostics into the high wavenumber regime (see, e.g., Refs. [1720]). Unfortunately, the list of observables does not include electron temperature and electrostatic potential fluctuations (or their cross phases). Thus, it is currently not possible to determine electron heat flux spectra directly. Instead, most high- $k$ diagnostics measure density fluctuation spectra. While the latter may serve as an indicator of the role of turbulence on sub-ion-gyroradius scales, little is known about their connection with electron heat flux spectra. Therefore, the present work is dedicated to an investigation of several experimentally accessible quantities as for instance density or frequency spectra by means of nonlinear gyrokinetic simulations covering both electron and ion spatio-temporal scales self-consistently. Our findings are supposed to shed light, in particular, on the link between large ETG-induced transport and recognizable features of the associated density fluctuations.

The present paper is organized as follows. In Section II, some background information on the nonlinear gyrokinetic simulations is provided, including the choice of physical and numerical parameters. In Section III, wavenumber spectra of the heat and particle fluxes are presented for three basic scenarios, two of which involve large ETG-induced contributions to the electron heat flux. Then, in Sections IV and V, these three cases are analyzed with respect to the resulting density and frequency spectra. In this context, it will become clear to what extent and how these experimentally observable quantities are related to the transport spectra. The paper closes with some conclusions in Section VI.

\section{SIMULATION DETAILS}

The tool employed for this study is the gyrokinetic turbulence code GENE [6, 21]. Although this code is able to treat magnetic field fluctuations and collisions, these effects are neglected here for simplicity. Moreover, the possibility to extract geometrical information for GENE from magnetohydrodynamic equilibria via the TRACER interface [22] is not used here. Instead, all simulations in this paper are performed in $\hat{s}$ - $\alpha$ geometry with $\alpha=0$, allowing for a relatively low resolution of 16 grid points in the $(z)$ direction parallel to the magnetic field. This, in turn, renders possible the use of up to $768 \times 384$ grid points in the radial $(x)$ and binormal $(y)$ directions which is essential for resolving electron gyroradius scales in simulation domains that are many ion gyroradii wide. Actually, to ensure an adequate coverage of the turbulent structures at long wavelengths, the perpendicular box size is chosen to be $\left(L_{x}, L_{y}\right)=\left(64 \rho_{s}, 64 \rho_{s}\right)$ where $\rho_{s}$ is the ion gyroradius at electron temperature given by $\rho_{s}=c_{s} / \Omega_{i}$ with ion sound speed $c_{s}=\sqrt{T_{e 0} / m_{i}}$ and ion Larmor frequency $\Omega_{i}=e B /\left(m_{i} c\right)$. In $\left(v_{\|}, \mu\right)$ velocity space, $32 \times 8$ grid points are used.

The physical parameters used in this work are variants of the Cyclone Base Case (CBC) values [23]. All simulations shown below employ a safety factor of $q=1.4$, a magnetic shear of $\hat{s}=0.8$, an inverse aspect ratio of $\epsilon=r / R=0.18$, and an equilibrium density/temperature ratio of $n_{i} / n_{e}=1$ and $T_{i} / T_{e}=1$, respectively. In contrast to the standard CBC scenario, two particle species (electrons and singly charged ions) are kept, both of them fully gyrokinetic. Since the computational effort roughly scales like $\left(m_{i} / m_{e}\right)^{3 / 2}$ (due to the scale separation in $x$, $y$, and $t$ ), we will work with a reduced mass ratio of $m_{i} / m_{e}=400$; still, each simulation requires of the order of 100, 000 CPUh.

In the following, we will focus primarily on three ba- 
sic scenarios for which we will discuss transport, density, and frequency spectra. They differ with respect to the normalized density and temperature gradients $R / L_{n}$ and $R / L_{T_{j}}(j=e, i)$ which may deviate from the nominal CBC values. The first one (A) with $R / L_{T_{i}}=R / L_{T_{e}}=$ 6.9 and $R / L_{n}=2.2$ reflects a physical situation in which the turbulence is driven (and very strongly) by ion temperature gradient (ITG) modes. Since the resulting value for the ion thermal diffusivity overestimates the experimentally determined one by almost two orders of magnitude [16], we furthermore investigate a case (B) with a reduced low- $k$ drive, characterized by $R / L_{T_{i}}=5.5$, $R / L_{T_{e}}=6.9$, and $R / L_{n}=0.0$. Here, the high- $k$ fluctuations, driven by ETG modes, contribute significantly to the total electron heat flux, and a scale separation between this transport channel and the remaining ones is clearly visible. Finally, we concentrate on a gradients choice (C) with $R / L_{T_{i}}=0.0, R / L_{T_{e}}=6.9$, and $R / L_{n}=0$, in which (only) trapped electron modes (TEMs) and ETG modes are driving the turbulence. This may happen, e.g., in plasmas with dominant electron heating or relatively high beta values. In the next section, we will provide simulation data for the resulting transport spectra in $k_{x}-k_{y}$ space. After that, density and frequency spectra will be discussed in Sections IV and V.

\section{HEAT AND PARTICLE FLUXES}

The electron and ion heat transport spectra in $k_{y}$ space for the three cases (A)-(C) have already been presented in Ref. [16]. In this context, it was found that for experimentally realistic ion heat (and particle) flux levels in the low- $k$ regime (corresponding to the latter two cases) and in the presence of unstable ETG modes, there tends to be a scale separation between electron and ion thermal transport. In contrast to the latter, the former may exhibit substantial or even dominant highwavenumber contributions carried by ETG modes and short-wavelength TEMs which are only relevant nonlinearly if ETG modes are unstable. Here, we would like to extend these analyses by also showing particle transport spectra and by decomposing the wavenumber contributions according to both $k_{y}$ and $k_{x}$. For this purpose, contour plots using logarithmically distributed colors are shown in Figs. 1 and 2.

As can be seen in Fig. 1, the ion heat flux spectra are always found to be dominated by wavelengths of the order of many ( $\gtrsim 10$ ) ion gyroradii, in good qualitative agreement with pure large-scale turbulence simulations (see, e.g., Ref. [24]). However, the electron heat flux spectra behave differently. Here, the contributions from shortwavelength turbulence increases with decreasing low- $k$ $\left(R / L_{T_{i}}\right)$ drive. Quantitatively, the high- $k_{y}\left(k_{y} \rho_{s}>1.0\right)$ fraction of the electron heat transport rises from $10.5 \%$ in case (A) to $42.3 \%$ in case (B) and finally reaches more than $50 \%$ in case $(\mathrm{C})$. In the radial direction, the $k_{x} \rho_{s}>1.0$ fraction in case (A) is $10.6 \%$ which is al-
(A) ions

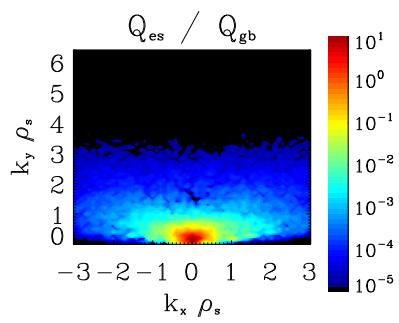

(B) ions

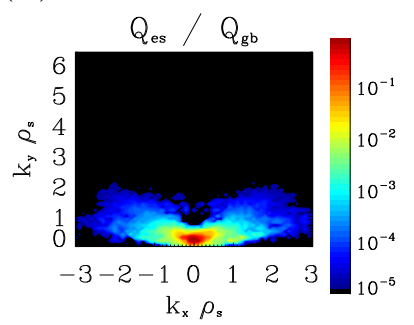

(C)
(A) electrons

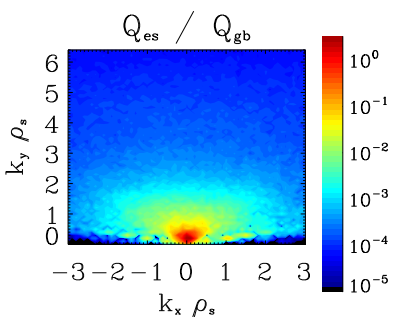

(B) electrons

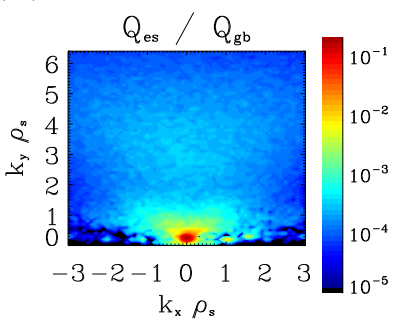

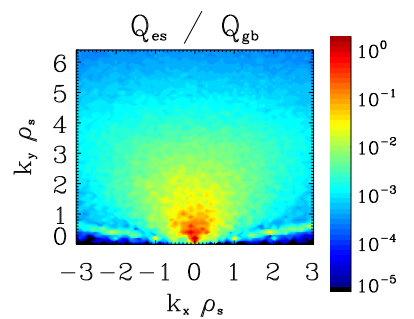

FIG. 1: (Color online) Time-averaged heat fluxes (normalized to $Q_{g b}=p_{0} c_{s} \rho_{s}^{2} / R^{2}$ ) vs radial and binormal wavenumbers for the multiscale simulations (A)-(C). In case (C), the ion heat flux is negligible and therefore not shown.

most identical to the respective $k_{y}$ fraction. However, in cases (B) and (C), the high- $k_{x}$ contributions amount to values around $30 \%$, therefore implying an anisotropic heat flux spectrum. The physical origin of these high$k$ anisotropies is most likely the existence of small-scale streamers $[6,7,16]$. This may be the case as long as the low- $k$ drive of the turbulence is of moderate strength. It is probably useful at this point to recall that case (A) is associated with unrealistically large ion heat fluxes, as has been mentioned before. Therefore, high- $k$ transport anisotropies are only to be expected for the other two cases which are, at the same time, to be considered more relevant. The particle fluxes, presented in Fig. 2, are directed inwards (describing a particle pinch) in cases (A) and $(\mathrm{B})$, but change sign in case (C) where ITG modes are not excited anymore. In all three situations, there are no significant high- $k\left(k \rho_{s}>1\right)$ contributions to the particle transport. This is in line with general expectations based on the fact that the ions become adiabatic at these scales.

Let us stress again that the three cases (A)-(C) represent three prototypical examples for two-scale turbulence. Case (A) corresponds to a system which is driven 
(A)

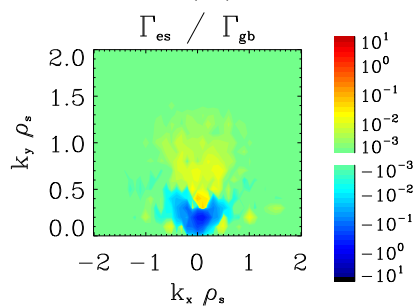

(B)
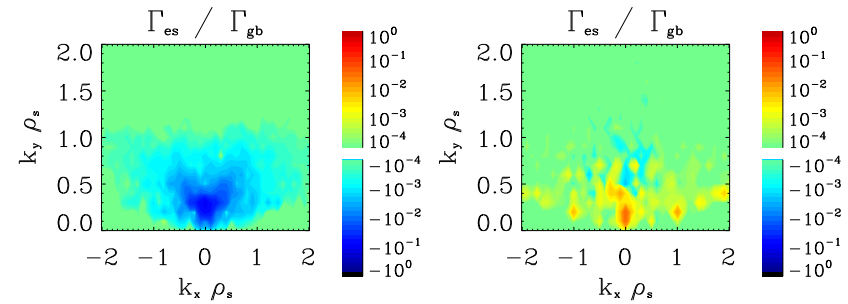

FIG. 2: (Color online) Time-averaged particle fluxes (normalized to $\left.\Gamma_{g b}=n_{0} c_{s} \rho_{s}^{2} / R^{2}\right)$ vs radial and binormal wavenumbers for the multiscale simulations (A)-(C).

very (actually, unrealistically) hard by ITG modes; case (B) describes a situation in which both ITG and ETG modes contribute to the transport; and in case (C), ITG modes are absent, leaving all the turbulent activity to TEMs and ETG modes. These basic scenarios, together with a few other cases, shall be analyzed in more detail in the remainder of this paper, with a particular focus on the resulting density spectra. Since the latter are directly accessible experimentally, it is of great interest to study how they are related to the transport spectra shown above.

\section{DENSITY SPECTRA}

Most core turbulence simulations up to date have been done for situations in which there was only one mode type, like an ITG mode or a TEM, driving the system. In these "pure" cases, the density fluctuation spectrum in the binormal direction, $S\left(k_{y}\right)=\left\langle|\tilde{n}(\mathbf{k}, \omega)|^{2}\right\rangle_{x, z, \omega}$ with $\langle\ldots\rangle$ denoting averages over quantities listed as indices, usually exhibits a maximum at $k_{y} \rho_{s} \sim 0.1-0.2$ whereas the radial spectra $S\left(k_{x}\right)=\left\langle|\tilde{n}(\mathbf{k}, \omega)|^{2}\right\rangle_{y, z, \omega}$ peak at wavenumbers close to zero. At higher wavenumbers, a power law $S\left(k_{x, y}\right) \propto k_{x, y}^{-a}$ is typically seen in both perpendicular directions. Here, the numerically determined scaling exponents are typically found to be in the range $a=3-5$ (see, e.g., Refs. [21, 25, 26]) which is consistent with the experimental findings $a \sim 3.5 \pm 0.5$ for medium wavenumbers $0.3 \lesssim k_{y} \rho_{s} \lesssim 1.0$ (see, e.g., [17] and references therein). For pure ETG turbulence, a similar behaviour has been observed in numerical simulations, where $\rho_{s}$ is replaced by the electron gyroradius $\rho_{e}[8,27]$.

Examples of highly resolved ITG and temperature gra- dient driven TEM turbulence simulations with linearly stable ETG modes are shown in Fig. 3 together with an ETG turbulence simulation employing a box size restricted to high- $k$ modes but retaining nonadiabatic ion dynamics. Here, the physical parameters are similar to those presented in Section II with the following exceptions. In the ITG simulation the gradients are $R / L_{T_{i}}=$ 6.92, $R / L_{T_{e}}=0.0, R / L_{n}=0.0$ whereas the TEM case uses $R / L_{T_{i}}=0.0, R / L_{T_{e}}=6.92, R / L_{n}=0.0$ and $T_{e} / T_{i}=3$. The settings for the ETG simulation are the nominal CBC values, therefore $R / L_{T_{i}}=R / L_{T_{e}}=6.92$ and $R / L_{n}=2.22$. Compared to the publications cited above, the binormal spectra are slightly flatter - which might be due to the use of different physical parameters or higher resolution. Furthermore, a bulge at high $k_{y}$ in the ITG density spectrum is observed which may be caused by a nonlinear excited ETG mode, an effect which has been reported before in Ref. [15]. Its absence in a simulation using the same parameters except for a finite Debye length of $\lambda_{D}=\rho_{s}$ supports this physical interpretation.

While "pure" turbulence simulations have the great advantage of minimizing the degree of complexity in performing and analyzing the runs, they usually represent idealized situations which are, in general, of limited value for direct comparisons with experimental findings. Thus, a step towards more realistic simulations involves the study of mixtures of two or more different turbulence types as they occur in the multiscale simulations mentioned before. The corresponding density spectra are presented in Fig. 4. With decreasing ion temperature gradient, a bulge at $k_{y} \rho_{s} \sim 2-5$ (corresponding to $\left.k_{y} \rho_{e} \sim 0.10-0.25\right)$ develops and becomes more and more pronounced. Since most of the ETG-induced transport is located in this wavenumber range and the radial spectrum does not show such a distinctive structure, we conclude that the modification of the binormal spectra is caused by the ETG modes, cf. Fig. 3. Outside of this wavenumber range, the observed power laws match more or less those known from pure turbulence simulations, except for case (A), where an unusually small exponent appears at the highest $k_{y}$ values. This is likely to be a numerical effect, however, which is expected to disappear when the perpendicular resolution is increased. In any case, a pronounced bulge in the binormal density spectrum as it occurs in case $(\mathrm{C})$ violates the often assumed isotropy of the density spectrum at high- $k$ modes. This can clearly be seen in Fig. 5 where the density spectra for cases (A) and (C) are plotted in $k_{x}-k_{y}$ space. While the former exhibits a more or less circular shape at $k_{x} \rho_{s} \sim k_{y} \rho_{s} \sim 1.0$, the latter displays an elongation in binormal direction in this mode range. As mentioned in Section III where a similar deformation has been observed in Fig. 1, this is due to the existence of ETG-scale streamers which seem to be hampered by the presence of strong ITG modes [16]. Of course, this anisotropy is not restricted to turbulence mixtures and may also be present in pure turbulence cases as can be seen by comparing the 
(a)

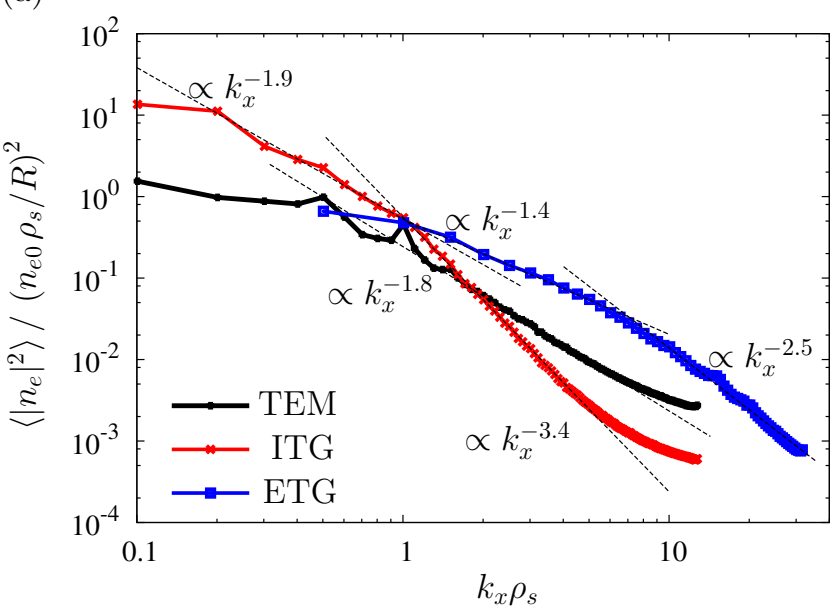

(b)

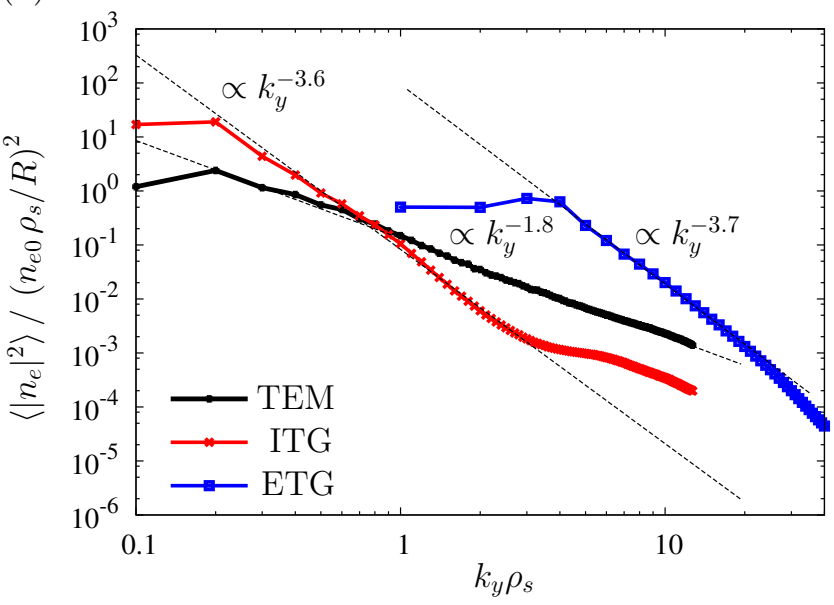

FIG. 3: (Color online) Squared electron density fluctuations for pure ITG, TEM, and ETG turbulence cases as a function of radial (a) and binormal (b) wavenumber, each averaged over the remaining directions and time. Since exact characteristics depend strongly on the chosen parameters, these results are only presented to demonstrate that the power law exponent is typically in the range of $2-4$, but not necessarily isotropic.

power law exponents in Fig. 3 in $k_{x}$ and $k_{y}$.

Such deviations from isotropy at short wavelengths should be taken into account when comparing numerical with experimental results. This is true, in particular, because in the latter case, the measured $k_{y}$ spectra often have $k_{x} \approx 0$ [17], while in simulations, it is common to average the squared amplitudes over the radial direction. In order to make comparisons easier, we have also evaluated the $k_{y}$ spectra for $k_{x}=0$. The corresponding binormal spectra are shown in Fig. 6. As expected, they differ from those presented in Fig. 3b and Fig. 4b, especially with respect to the power law exponents. One now finds exponents up to $a \sim 5$, and if a fit is applied to the range $4 \lesssim k_{y} \rho_{s} \lesssim 7$ in the pure ETG turbulence case, one even arrives at $a=7.4$. These values are quite close to (a)

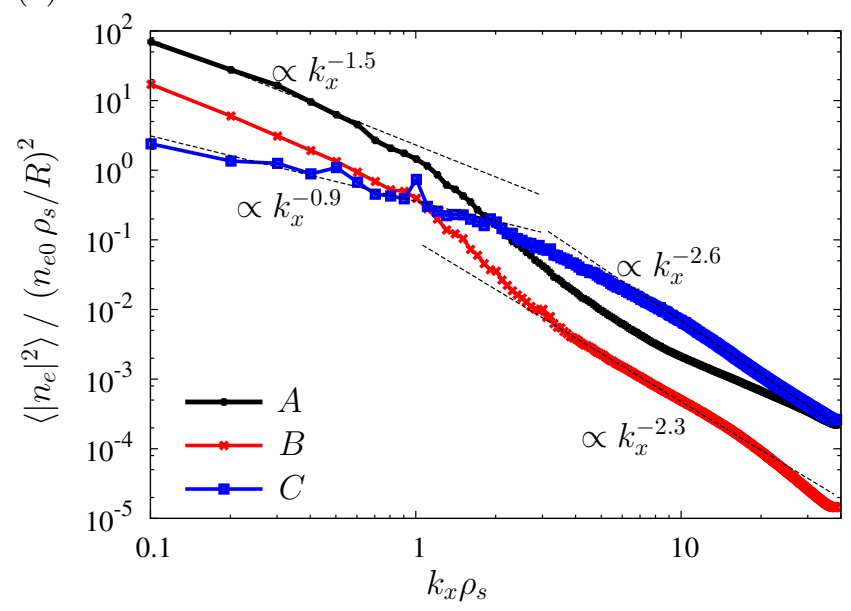

(b)

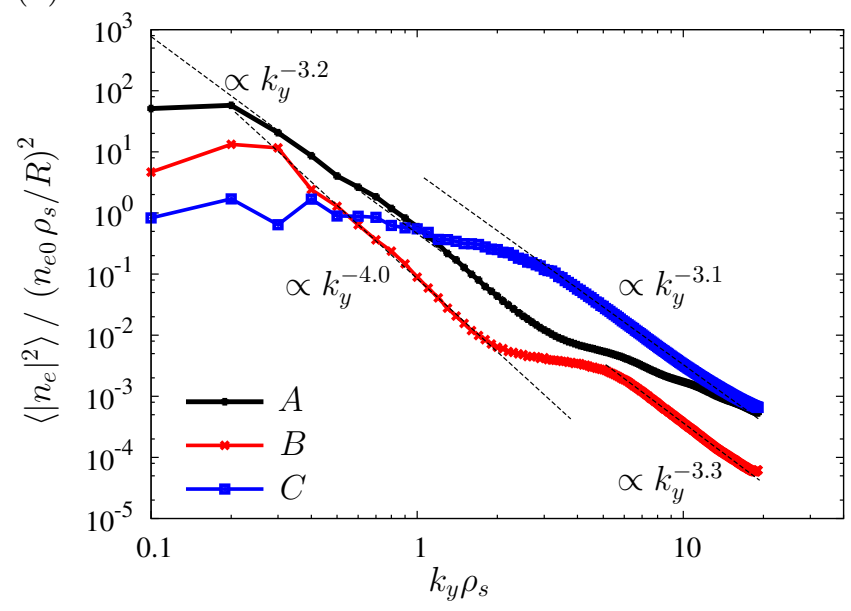

FIG. 4: (Color online) Squared electron density fluctuations averaged over the parallel and radial direction and time for turbulence mixtures with $R / L_{T_{e}}=6.9$ and (A) $R / L_{T_{i}}=6.9$, (B) $R / L_{T_{i}}=5.5$, and (C) $R / L_{T_{i}}=0.0$.

the experimental findings [17] where $a \sim 3.5$ was found at low- $k$, and $a \sim 6.5-7$ in the high- $k$ regime. Such characteristics are actually in good qualitative agreement with those of case $(\mathrm{C})$, but the power law exponents do not match. One finds $a \sim 1.9$ at $0.15<k_{y} \rho_{s}<2$ and $a \sim 5.0$ at $4<k_{y} \rho_{s}<10$. The inclusion of Debye shielding effects might reduce the difference since they may steepen the spectrum at high wavenumbers (see, e.g., Ref. [27]). A change of plasma parameters or magnetic geometry may also lead to better agreement. Similar arguments apply to the radial direction where Gurchenko and coworkers report a power law transition from $a \sim 2.5$ to $a \sim 6.2$ at $k_{y} \rho_{s} \sim 9$ [28].

Concluding this section, we would like to emphasize that a signature of strong ETG activity is that it tends to flatten the density spectra in the $k_{y} \rho_{e} \gtrsim 0.1$ region. (Note that for a realistic mass ratio of $m_{i} / m_{e}=1836$ or $m_{i} / m_{e}=3670$, this corresponds to $k_{y} \rho_{s} \gtrsim 4$ and 
(A)

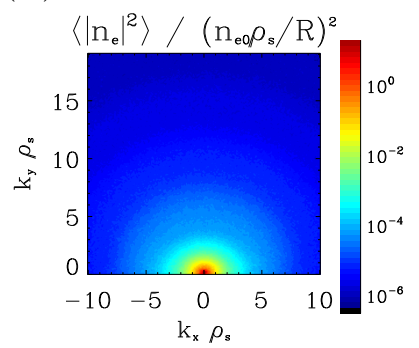

(C)

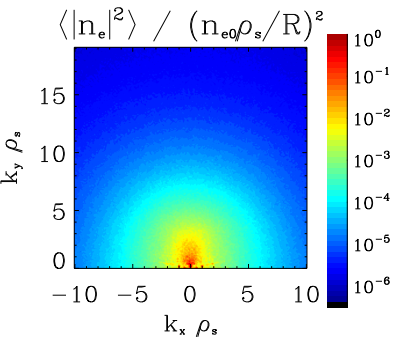

FIG. 5: (Color online) Squared electron density fluctuations as function of $k_{x}$ and $k_{y}$ from the multiscale simulations (A) and $(\mathrm{C})$.

(a)

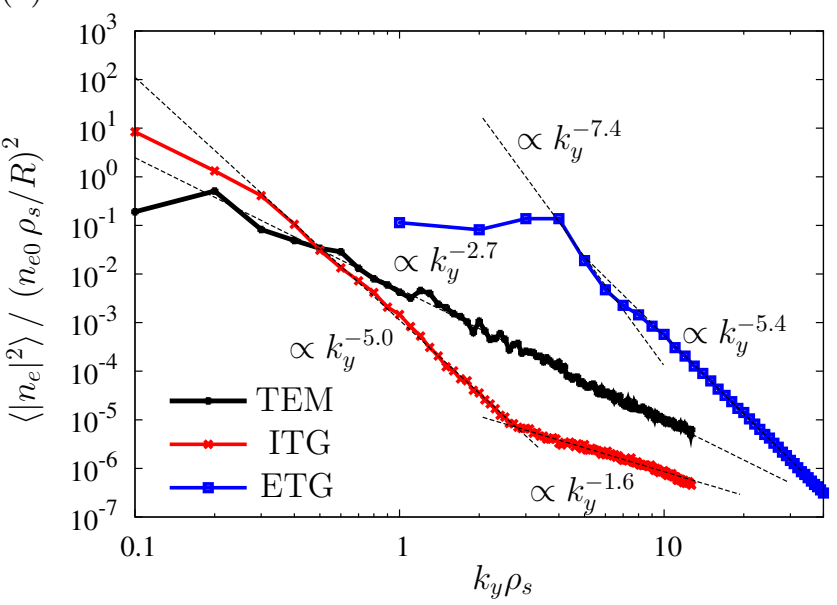

(b)

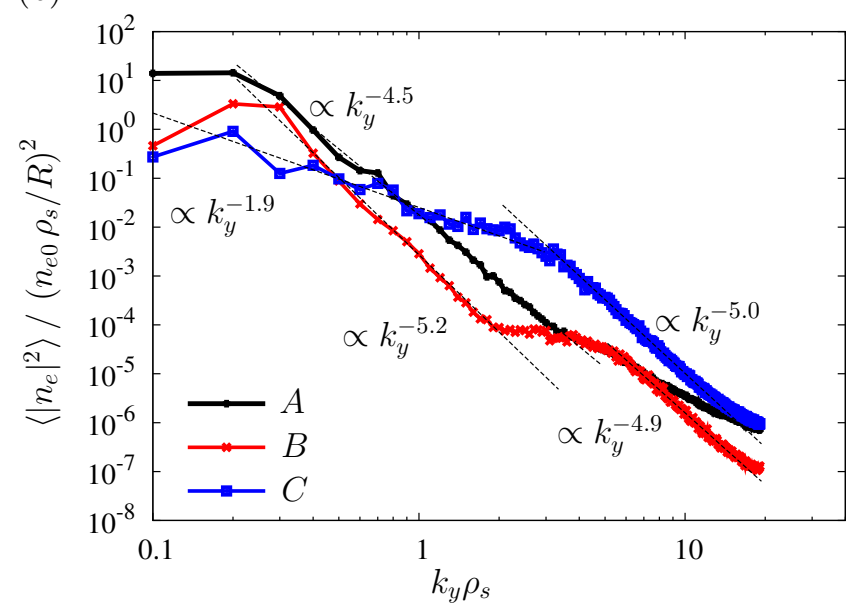

FIG. 6: (Color online) Squared electron density fluctuations evaluated at $k_{x} \rho_{s}=0$ and averaged over the parallel direction and time for (a) pure turbulence cases, cf. Fig. 3b, and (b) turbulence mixtures, cf. Fig. 4b. (a)

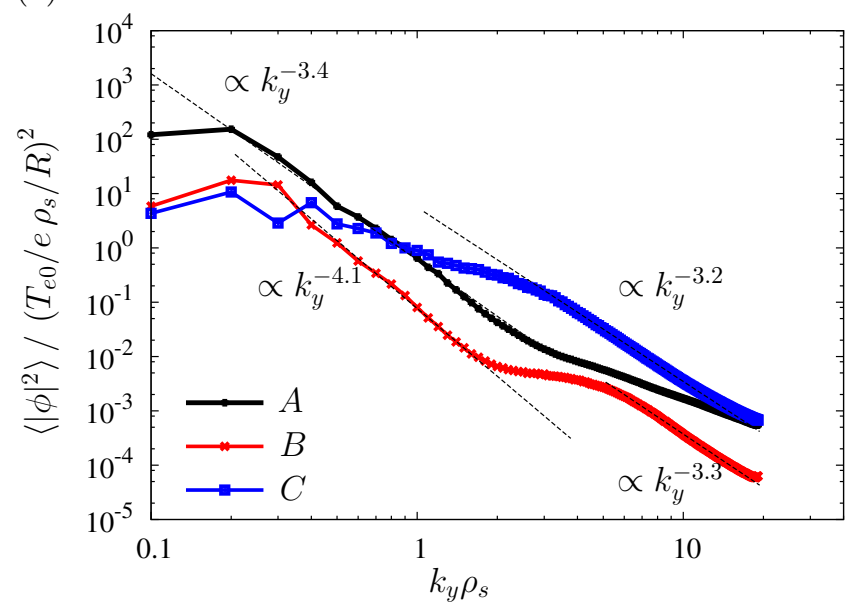

(b)

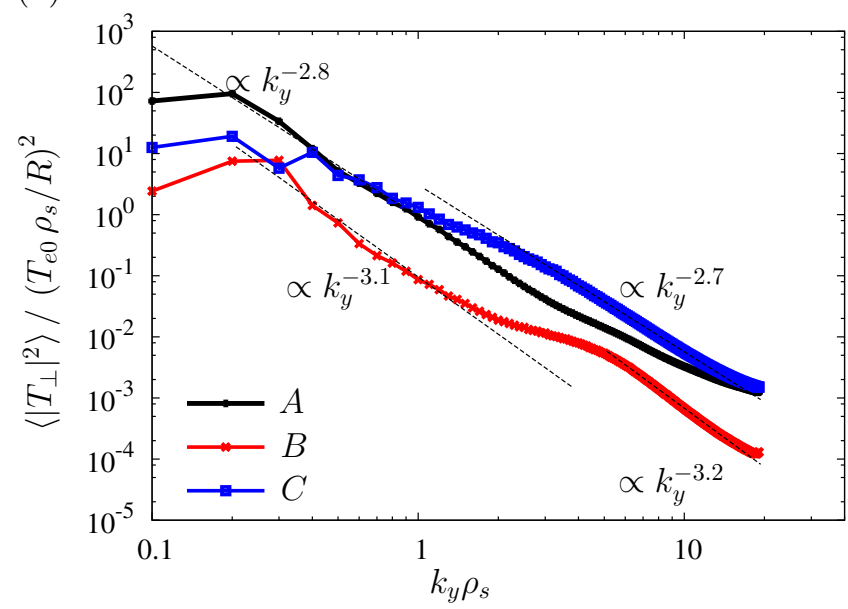

FIG. 7: (Color online) Squared electrostatic potential (a) and perpendicular temperature (b) fluctuations averaged over the radial and parallel direction and time for the multiscale simulations (A)-(C).

$k_{y} \rho_{s} \gtrsim 6$, respectively.) If the long-wavelength dynamics is dominated by ITG modes, the fall-off up to that point will still be substantial, however, and presumably no high- $k$ peaks are to be expected. Nevertheless, the ETG-induced contributions to the total electron heat flux can be large since most of it is driven by the positive correlations between fluctuations of the electrostatic potential and the electron temperature, both of which tend to decay more slowly than the density fluctuations. The respective spectra of these quantities are shown in Fig. 7 - but they cannot be measured in current experiments.

\section{FREQUENCY SPECTRA AND PHASE VELOCITIES}

Another turbulence characteristics which is rather accessible experimentally is a (nonlinear) spectrum of fre- 
(a)

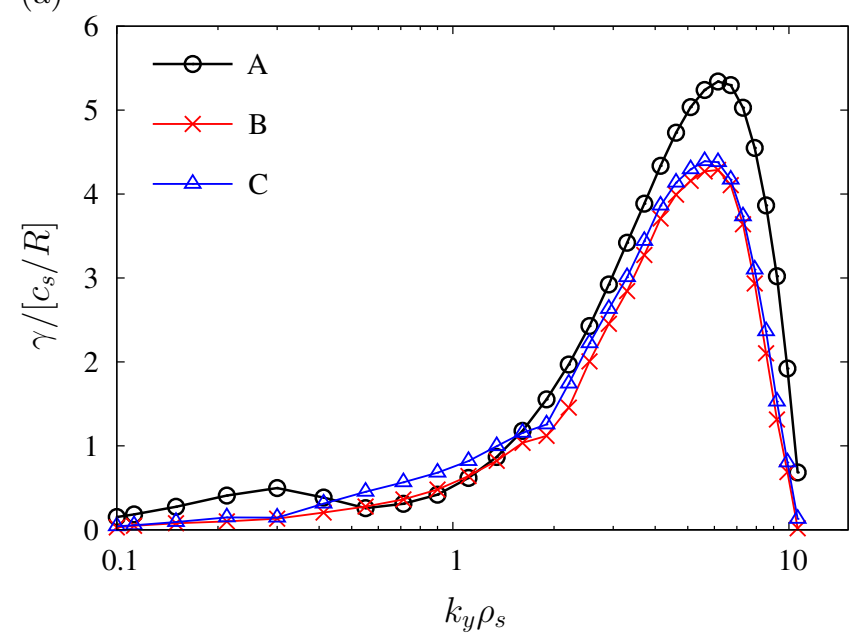

(b)

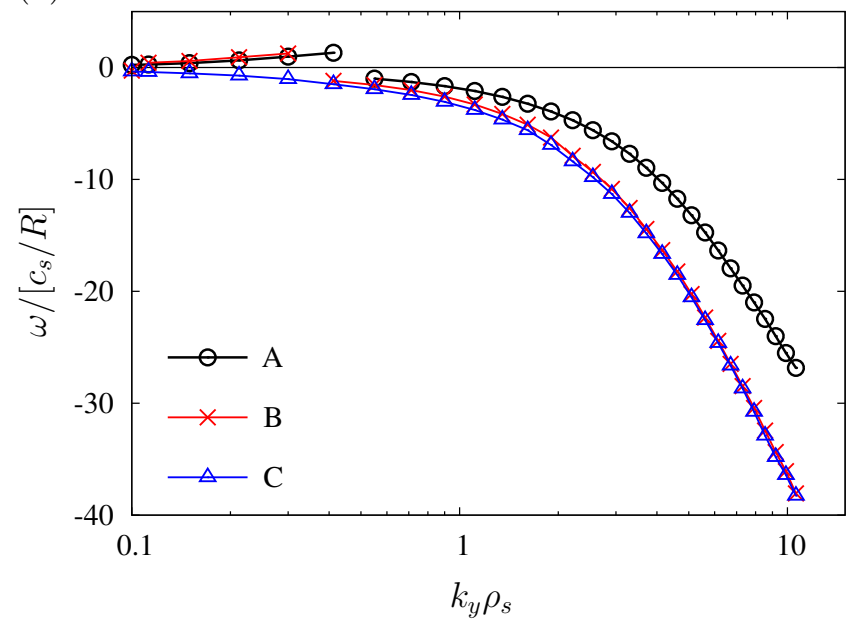

FIG. 8: (Color online) Linear growth rates (a) and real frequencies (b) at $k_{x} \rho_{s}=0$ vs. binormal wavenumber using (a) $R / L_{T_{i}}=R / L_{T_{e}}=6.9, R / L_{n}=2.2$, (b) $R / L_{T_{i}}=5.5$, $R / L_{T_{e}}=6.9, R / L_{n}=0$, and (c) $R / L_{T_{i}}=0, R / L_{T_{e}}=6.9$, $R / L_{n}=0$.

quencies or phase velocities. Fortunately, as we will see, these quantities are often closely linked to the respective linear quantities, such that one can infer relevant information already from rather inexpensive linear gyrokinetic simulations. However, nonlinear effects may change the dominant mode within a certain $k$ range with respect to the linear expectations. Such phenomena have to be taken into account when attempting to compare results from experiments and simulations.

According to the growth rate and frequency spectra corresponding to the multiscale simulations (A)-(C), as presented in Fig. 8, we see that different turbulence types are expected to dominate in different wavenumber regimes. This is most pronounced in case $(\mathrm{A})$ where a steep ion temperature gradient excites ITG modes which can be identified by a positive sign in real frequency and dominate up to a binormal wavenumber of $k_{y} \rho_{s} \sim 0.4$.

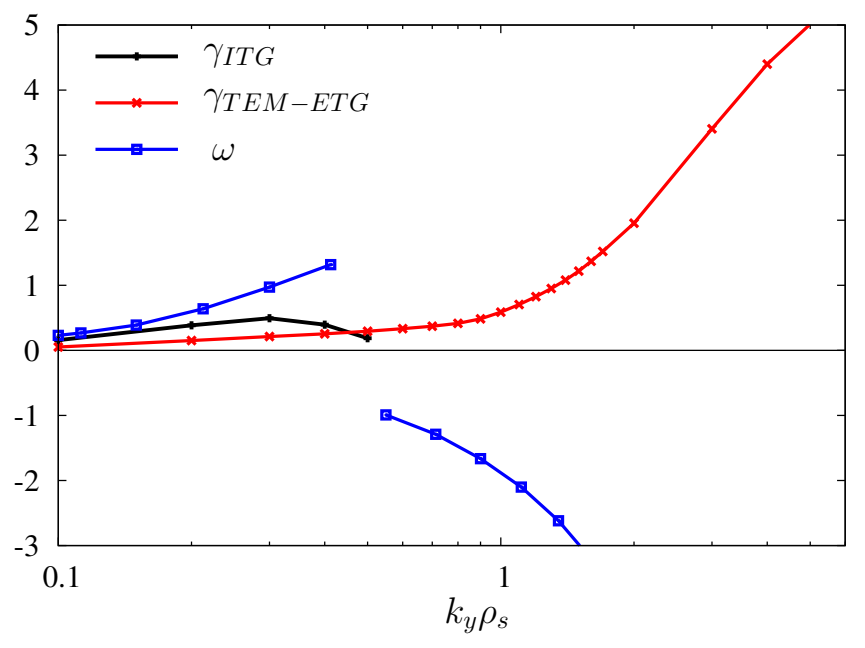

FIG. 9: (Color online) Linear growth rates of dominant and first subdominant mode and dominant real frequency at $k_{x} \rho_{s}=0$ in $c_{s} / R$ in the small up to medium $k_{y}$ range for the multiscale simulation $(\mathrm{A})$.

The abrupt change of sign in real frequency at higher wavenumbers suggests that trapped electron modes drifting in the electron diamagnetic direction take over before they transition into ETG modes. The latter can be clearly identified by their spatio-temporal separation compared to TEM and ITG modes which is given by the square root of ion to electron mass ratio, here 20 . However, this plot only shows the most dominant mode for each wavenumber while, in general, several unstable modes may exist at the same wavenumber. In particular, if the strongest subdominant mode is very close to the dominant one, it may alter the nonlinear behavior significantly. Therefore, the GENE code has been recently extended to also operate as an eigenvalue solver, enabling the calculation of subdominant modes [29]. The dominant and first subdominant mode for case (A) are presented in Fig. 9. Due to the real frequency's sign of the dominant mode the highest growth rate at $k_{y} \rho_{s}<0.5$ can easily be assigned to an ITG mode. At higher wavenumbers, a mode with negative frequency takes over, which may be labelled a TEM-ETG mode since it smoothly transitions from a low- $k$ TEM to a high- $k$ ETG mode. Such a transition is not surprising, given that all kinds of microinstabilities may be connected to each other [29].

Now, it is interesting to investigate whether and how the observed change from ITG to TEM-ETG modes is modified in the presence of nonlinear effects. In Fig. 10, the linear and nonlinear real frequencies calculated by using the approximation

$$
\omega \approx \Im\left[\log \left(\frac{\phi(t)}{\phi(t-\Delta t)}\right)\right] / \Delta t
$$

are presented. Given a reasonably long (linear) simulation time, Eq. (1) turns out to provide very reliable results. Nonlinearly, the approximation has to be handled with care, however, especially if two or more modes 
(A)

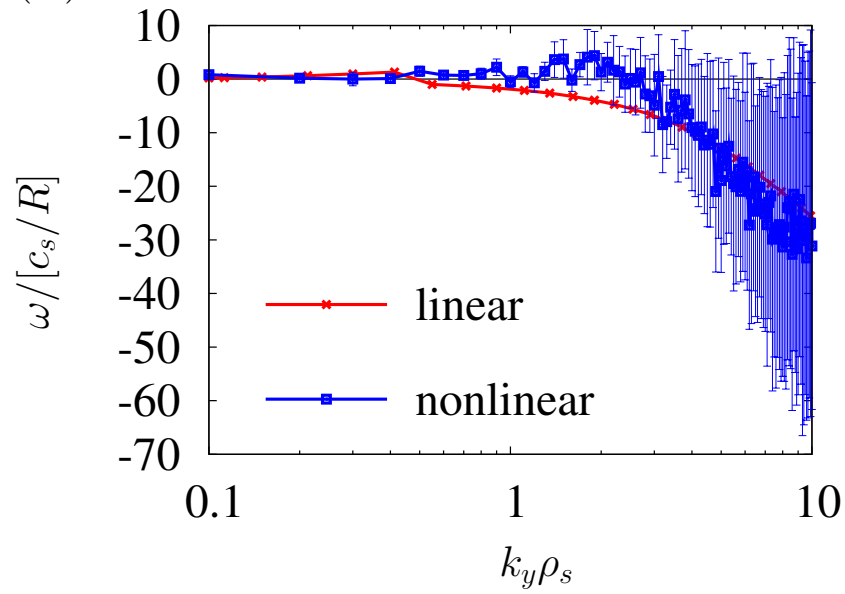

(B)

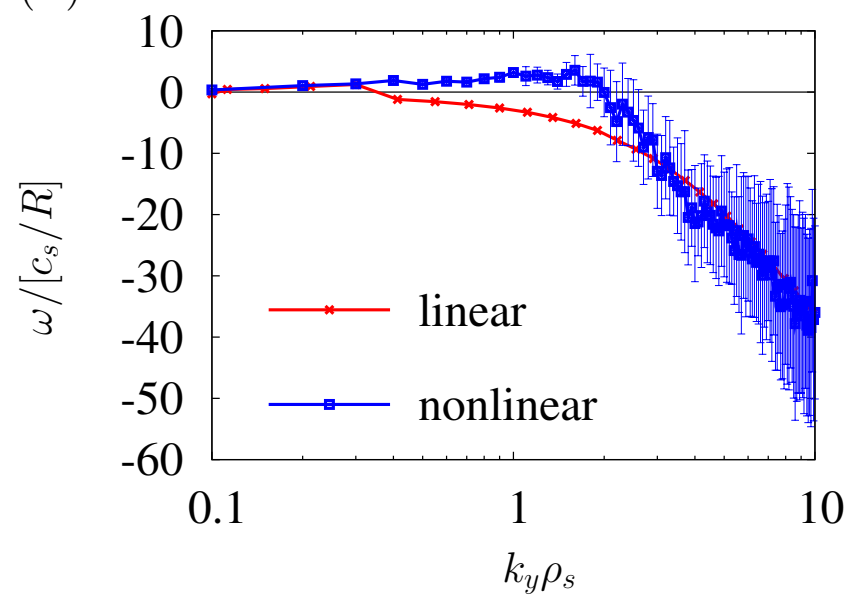

(C)

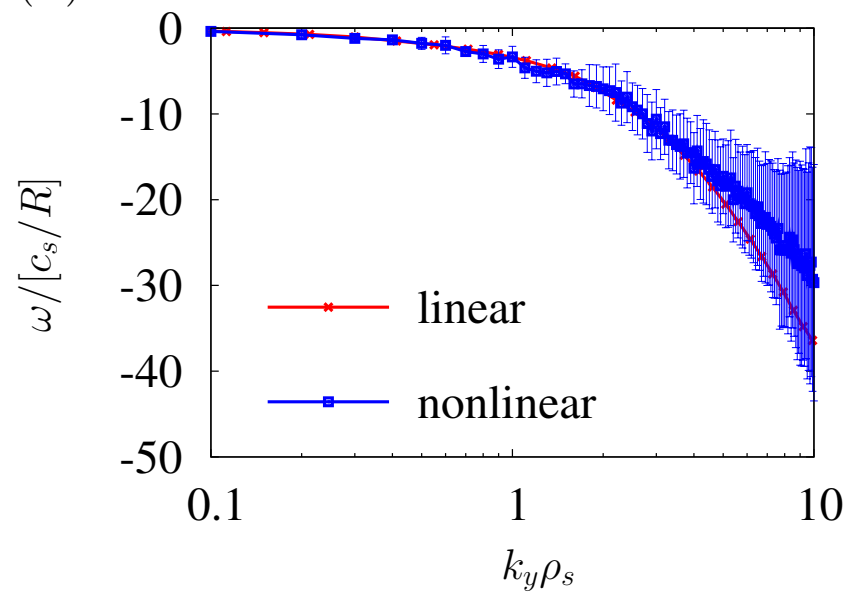

FIG. 10: (Color online) Dominant real frequency defined as median of Eq. (1) at $k_{x} \rho_{s}=0$ for the multiscale simulations (A)-(C) with and without consideration of the nonlinearity. The error bars denote the standard deviations. are strongly excited at the same wavenumber. In that case, the resulting value is not necessarily identical with the dominant real frequency but closely linked to the first moment of the frequency spectrum. On the other hand, Eq. (1) usually yields a robust and easy accessible quantity which at least allows to determine, e.g., whether a certain wavenumber is influenced more by ITG or by TEM-ETG modes. In Fig. 10, this type of study reveals some surprises. While nonlinear and linear frequencies agree well over a significant region in $k_{y}$ space in simulation (C), both simulations with unstable ITG modes $(\mathrm{A}, \mathrm{B})$ show differences when the ITG mode becomes linearly subdominant at $k_{y} \rho_{s} \approx 0.4$. Here, the real frequency stays predominantly positive up to $k_{y} \rho_{s} \sim 1.5$ thus exceeding even the wavenumber where ITG modes are linearly stabilized by almost a factor of 2 . At smaller scales, the nonlinear behavior reflects the linear one again to a good approximation. Furthermore, the standard deviations are included in Fig. 10 as error bars. With increasing wavenumber they become larger; thus, it is more difficult to assign a certain frequency to small-scale fluctuations. This may, in part, be due to cross-scale interactions with large-scale turbulence $[9,16]$. Nevertheless, in all cases shown, the existence of ETG turbulence at high wavenumbers is clearly reflected in the frequency spectra. The respective phase velocities are typically up to a few $c_{s} \rho_{s} / R$.

\section{CONCLUSIONS}

There is significant experimental and theoretical evidence which suggests that ETG modes may yield substantial or even dominant high-wavenumber contributions to the electron heat transport under certain conditions, e.g., in plasmas with dominant electron heating, relatively high beta values, substantial equilibrium $\mathbf{E} \times \mathbf{B}$ shear, and transport barriers. Motivated, in particular, by recent experimental advances in the area of high- $k$ density fluctuation diagnostics, we carried out gyrokinetic turbulence simulations with the GENE code, covering both ion and electron spatio-temporal scales selfconsistently, and analyzed them with respect to several experimentally accessible quantities like density and frequency spectra.

We found that in contrast to "pure" ITG or TEM turbulence cases, multiscale simulations involving unstable ETG modes tend to exhibit a flat region in the binormal wavenumber spectrum of the density fluctuations at $k_{y} \rho_{e} \gtrsim 0.1$. At both longer and shorter wavelengths, power law decays are observed which are more or less in line with respect to earlier, single-scale simulations in terms of the measured exponents. In the case of a TEMETG turbulence mixture (with stable ITG modes), we found a remarkable level of agreement with recent experimental findings, claiming that the power law exponent becomes much larger at $k_{y} \rho_{s} \gtrsim 1$. However, the results do not match quantitatively, most probably because sev- 
eral potentially important physical effects (like collisions, magnetic fluctuations, real geometry, or a finite Debye length) were neglected here for simplicity. We would also like to point out that most experimental measurements were done close to the edge, while our multiscale simulations employed typical core parameters. In addition, increasing the mass ratio to realistic values would lead to a further separation of ion and electron scales and therefore might alter some of the results quantitatively.

Nevertheless, our qualitative findings are expected to remain valid, in particular the fact that high- $k$ modes may contribute significantly to the electron heat transport although the density spectra might exhibit a rather fast decay. On the other hand, the experimental detection of a flat region in the binormal wavenumber spectrum of the density fluctuations at around $k_{y} \rho_{e}>0.1$ would be good evidence for the existence of strong ETG activity. At the same time, our simulations show that measurement of frequencies or phase velocities at short wavelengths can be used to establish the existence of ETG turbulence.

\section{Acknowledgements}

The authors wish to thank F. Merz for help with the Gene code, as well as C. Tröster, T. Rhodes, D. Smith, and X. Garbet for helpful discussions. Moreover, we thank the DEISA Consortium (www.deisa.eu), co-funded through EU FP6 projects RI-508830 and RI-031513, for support within the DEISA Extreme Computing Initiative. The computations were performed at the Leibniz and Garching Computing Centers.
[1] B. W. Stallard, C. M. Greenfield, G. M. Staebler, C. L. Rettig, M. S. Chu, M. E. Austin, D. R. Baker, L. R. Baylor, K. H. Burrell, J. C. DeBoo, J. S. deGrassie, E. J. Doyle, J. Lohr, G. R. McKee, R. L. Miller, W. A. Peebles, C. C. Petty, R. I. Pinsker, B. W. Rice, T. L. Rhodes, R. E. Waltz, and L. Zeng, Phys. Plasmas 6, 1978 (1999).

[2] S. M. Kaye, F. M. Levinton, D. Stutman, K. Tritz, H. Yuh, M. G. Bell, R. E. Bell, C. W. Domier, D. Gates, W. Horton, J. Kim, B. P. LeBlanc, N. C. Luhmann Jr., R. Maingi, E. Mazzucato, J. E. Menard, D. Mikkelsen, D. Mueller, H. Park, G. Rewoldt, S. A. Sabbagh, D. R. Smith and W. Wang, Nucl. Fusion 47, 499 (2007).

[3] T. L. Rhodes, W. A. Peebles, M. A. Van Zeeland, J. S. deGrassie, R. V. Bravenec, K. H. Burrell, J. C. DeBoo, J. Lohr, C. C. Petty, X. V. Nguyen, E. J. Doyle, C. M. Greenfield, L. Zeng, and G. Wang, Phys. Plasmas 14, 056117 (2007).

[4] T. Zhang, X. Gao, Y. Li, and J. Zhao, Phys. Lett. A 372, 4705 (2008).

[5] E. Mazzucato, D. R. Smith, R. E. Bell, S. M. Kaye, J. C. Hosea, B. P. LeBlanc, J. R. Wilson, P. M. Ryan, C. W. Domier, N. C. Luhmann, Jr., H. Yuh, W. Lee, and H. Park Phys. Rev. Lett 101, 075001 (2008).

[6] F. Jenko, W. Dorland, M. Kotschenreuther, and B. N. Rogers, Phys. Plasmas 7, 1904 (2000).

[7] W. Dorland, F. Jenko, M. Kotschenreuther, and B. N. Rogers, Phys. Rev. Lett. 85, 5579 (2000).

[8] F. Jenko and W. Dorland, Phys. Rev. Lett. 89, 225001 (2002).

[9] F. Jenko, J. Plasma Fusion Res. 6, 11 (2004).

[10] Y. Idomura, Phys. Plasmas 13, 080701 (2006).

[11] N. Joiner, D. Applegate, S. C. Cowley, W. Dorland, and C. M. Roach, Plasma Phys. Control. Fusion 48, 685 (2006).

[12] W. M. Nevins, J. Candy, S. Cowley, T. Dannert, A. Dimits, W. Dorland, C. Estrada-Mila, G. W. Hammett, F. Jenko, M. J. Pueschel, and D. E. Shumaker, Phys. Plasmas 13, 122306 (2006).

[13] A. Bottino, A. G. Peeters, R. Hatzky, S. Jolliet, B. F. McMillan, T. M. Tran, and L. Villard, Phys.
Plasmas 14, 010701 (2007).

[14] J. Candy, R. E. Waltz, M. R. Fahey and C. Holland, Plasma Phys. Control. Fusion 49, 1209 (2007).

[15] R. E. Waltz, J. Candy, and M. Fahey, Phys. Plasmas 14, 056116 (2007).

[16] T. Görler and F. Jenko, Phys. Rev. Lett 100, 185002 (2008).

[17] P. Hennequin, R. Sabot, C. Honoré, G. T. Hoang, X. Garbet, A. Truc, C. Fenzi and A. Quéméneur, Plasma Phys. Control. Fusion 46, B121 (2004).

[18] D. R. Smith, E. Mazzucato, T. Munsat, H. Park, D. Johnson, L. Lin, C. W. Domier, M. Johnson, and N. C. Luhmann, Jr., Rev. Sci. Instrum. 75, 3840 (2004).

[19] T. L. Rhodes, W. A. Peebles, X. Nguyen, M. A. VanZeeland, J. S. deGrassie, E. J. Doyle, G. Wang, and L. Zeng, Rev. Sci. Instrum. 77, 10E922 (2006).

[20] C. Tröster, Ph.D. thesis, Ludwig-MaximiliansUniversität München, 2008.

[21] T. Dannert and F. Jenko, Phys. Plasmas 12, 072309 (2005).

[22] P. Xanthopoulos and F. Jenko, Phys. Plasmas 13, 092301 (2006).

[23] A. M. Dimits, G. Bateman, M. A. Beer, B. I. Cohen, W. Dorland, G. W. Hammett, C. Kim, J. E. Kinsey, M. Kotschenreuther, A. H. Kritz, L. L. Lao, J. Mandrekas, W. M. Nevins, S. E. Parker, A. J. Redd, D. E. Shumaker, R. Sydora, and J. Weiland, Phys. Plasmas 7969 (2000).

[24] J. E. Kinsey, R. E. Waltz, and J. Candy, Phys. Plasmas 13, 022305 (2006).

[25] F. Jenko and B. Scott, Phys. Plasmas 6, 2705 (1999).

[26] S. V. Henriksson, S. J. Janhunen, T. P. Kiviniemi, and J. A. Heikkinen, Phys. Plasmas 13, 072303 (2006).

[27] F. Jenko and A. Kendl, Phys. Plasmas 9, 4103 (2002).

[28] A. D. Gurchenko, E. Z. Gusakov, A. B. Altukhov, A. Yu. Stepanov, L. A. Esipov, M. Yu. Kantor, D. V. Kouprienko, V. V. Dyachenko and S .I. Lashkul, Nucl. Fusion 47, 245 (2007).

[29] M. Kammerer, F. Merz, and F. Jenko, Phys. Plasmas 15, 052102 (2008). 